\title{
Studies on Evaluation Index System for the Traffic Impact Evaluation Report Via the Triangular Fuzzy Number Hierarchical Method
}

\author{
$\mathrm{H} \mathrm{B} \mathrm{XU}{ }^{*}$ \\ ${ }^{1}$ Road Administration and Facilities Department, Traffic Police Corps, Shanghai Public Security Bureau, Shanghai 201204, China
}

\begin{abstract}
Traffic impact evaluation is quantitatively analysis aimed at new traffic demand after the construction project completed and put into use or the using change of urban land ${ }^{[1]}$. Traffic impact evaluation was first implemented locally in large cities of China, and then the relevant departments issued formal technical standards ${ }^{[2]}$. After 20 years of accumulation and development, almost all cities have formulated technical standards and management, implementation methods, lots of academic achievements and innovative experience were achieved. It has become an important basis of urban construction project planning, management and decision-making, also an important joint between technology and management [1]. Since 2010, the number of traffic impact evaluation projects has increased substantially. The implementation of projects and the standardization of projects report are the key factors for the sustainable development of the traffic impact evaluation market, and also affect the strategic deployment of urban transportation. In this paper, the triangular fuzzy number hierarchical method is used ${ }^{[3]}$. Based on the questionnaire data of specialist, the first level and second level index and respective weight distribution of evaluation index system for traffic impact evaluation report were obtained. The weight of index was adjusted through consistency judgment for assuring the rationality of index weigh. Evaluation index system was established based on the above mentioned studies, and the reference basis was provided for the evaluation of traffic impact evaluation report in the future.
\end{abstract}

\section{Introduction}

In recent years, with the acceleration of urbanization construction in China, the contradiction between urban traffic demand and traffic supply has become prominent. The disorderly and high-intensity development has resulted in unbalanced of regional transport infrastructure carrying capacity and traffic demand, deteriorating urban traffic environment and overcrowded roads. In order to promote the rationalization of urban development and construction, realize the coordinated development of land use and transportation, and build a smooth city, the relevant departments put forward the requirement of traffic impact evaluation for construction projects ${ }^{[1]}$. In 2010, the Ministry of Housing and Urban-Rural Construction officially promulgated the Technical Standards for Traffic Impact Evaluation of Construction Projects. Traffic impact evaluation is quantitatively analysis aimed at new traffic demand after the construction project completed and put into use or the using change of urban land. At present, almost all cities have formulated technical standards and management, implementation methods, lots of academic achievements and innovative experience were achieved. It has become an important basis of urban construction project planning, management and decision-making, also an important joint between technology and management ${ }^{[1]}$.

Traffic impact assessment studies local, individual and actual traffic problems, proposes internal, external and regional traffic solutions, and defines the traffic conditions for construction or program implementation. From the technical point of view, it extends the urban traffic planning from macro to micro, from vague to concrete; from the urban management point of view, it provides a basis for project decision-making, regional traffic construction and traffic solutions ${ }^{[1]}$. Scholars have analyzed the traffic impact of various types of construction projects, such as urban construction projects, commercial and trade construction projects, residential projects, industrial projects and so on. The research target also includes evaluation methods, evaluation models, evaluation periods and post-evaluation models ${ }^{[4-}$ 6]. However, the review and quantitative assessment of traffic impact evaluation reports has not been involved. Establishing the evaluation system of traffic impact evaluation reports can provide criteria and basis for the quality evaluation of traffic impact evaluation reports, and provide methods and basis for the optimization and improvement of future reports.

Because the evaluation of traffic impact evaluation reports is a multi-level, multi-factor, wide-ranging and

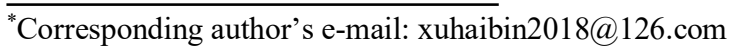


standard-ambiguous research problem, and the fuzzy hierarchical comprehensive evaluation and decisionmaking strategy is a very effective multi-factor decisionmaking method to make a comprehensive evaluation of the things affected by many factors. It can evaluate and rank the evaluation objects according the comprehensive score. The triangular fuzzy number hierarchical method is chosen ${ }^{[3,7]}$ in this paper, the weights of the first and second level index which affect the traffic impact evaluation reports were calculated, the evaluation index system and model were established.

\section{Materiel and method}

\subsection{Data sources}

In the evaluation of the traffic impact assessment report, the comprehensiveness of basic data, traffic volume prediction, authenticity and reliability of the breakdown data, traffic organization design, reporting function and report compilation are five very important evaluation indicators. Through consultation with the management of the Transport Commission, the five factors are selected as the first-level indicators, and the initial second-level indicators under each first-level indicator are listed. In order to improve the reliability of decision-making, triangular fuzzy numbers are added and expert consultation papers are integrated for analysis.

\subsection{Data analysis methods}

\subsubsection{Construction of Fuzzy Judgment Matrix}

According to the requirement of the overall target, the fuzzy judgment matrix of the evaluation is obtained by comparing the two factors. The fuzzy judgment matrix is noted ${ }^{[3]}$.

$$
R=\left(r_{i j}\right)_{n} \times n
$$

In the equation $1, \mathrm{n}$ is the number of evaluation index, $r_{i j}=\left(l_{i j}, m_{i j}, u_{i j}\right)$ is a triangular fuzzy number, $l_{i j}, u_{i j}$ are the upper and lower bounds, $m_{i j}$ is measurement scale number, the 0.1-0.9 scale method is adopted in this study ${ }^{[3]}$, which is more convenient and more reflective of real estimation. The scale of triangular fuzzy number given by experts is referred to Table 1. The data are averaged and the fuzzy judgment matrix is obtained.

Table 1 . The scale of triangular fuzzy number.

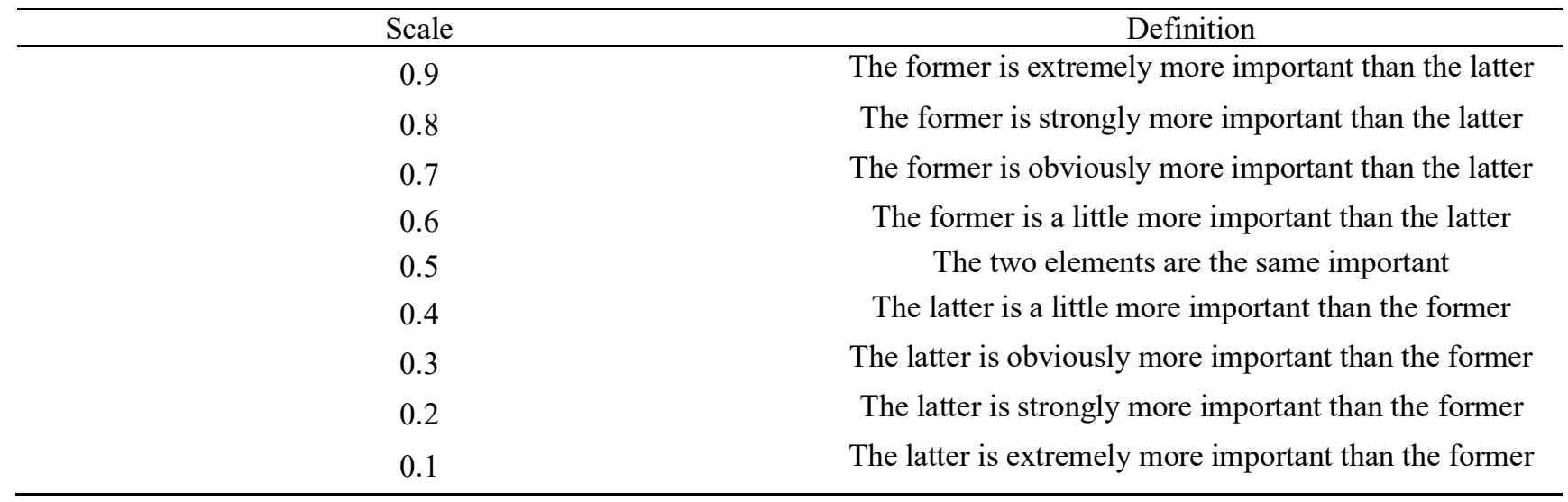

\subsubsection{Conversion of Fuzzy judgement Matrix}

Since the triangular matrix given by experts is fuzzy, it needs to be transformed into an accurate number judgment matrix firstly. after the carrying out of consistency test, the judgment matrix is normalized to get the weight of all levels of index. In this paper, Xiao Y. ${ }^{[8]}$ method is used to construct an accurate judgment matrix by obtaining the expected values of triangular fuzzy numbers.

$$
m_{i j}^{\prime}=\frac{1}{2(1+N)} l_{i j}+\frac{N+2 N M+M}{2(1+N)(1+M)} m_{i j}+\frac{1}{2(1+M)} u_{i j}
$$

Where, $\mathrm{N}$ denotes how many times the probability of that the result is $m_{i j}$ than $l_{i j}, \mathrm{M}$ denotes how many times the probability of the result is $m_{i j}$ than $u_{i j}$. In this study, $\mathrm{M}=\mathrm{N}=2$.

\subsubsection{Consistency test}

In order to test the consistency of the accurate judgment matrix, the key point of matrix consistency which is the additive consistency index $\rho$, the larger the $\rho$ value is, the worse of the consistency is obtained. In this study, the $\varepsilon=0.2$ is set. If the consistency index satisfies the condition of $\rho \leq \varepsilon$, the judgment matrix has satisfactory consistency, contrarily the judgment matrix does not satisfy the consistency requirement, then we need to adjust the matrix factor to achieve $\mathrm{it}^{[3]}$.

$$
\rho=\frac{2}{n(n-1)(n-2)} \sum_{i=1}^{n-1} \sum_{\substack{j=i+1 \\ k=1 \\ k \neq i, j}}^{n} \sum_{i j}^{n}-\left(m_{i k}^{\prime}+m_{k j}^{\prime}-0.5\right) \mid
$$




\subsubsection{Establishing of normalized weight value of evaluation index}

The weight of each index is calculated by the judgment matrix after consistency test, and the weight vector of each index is obtained by normalizing the judgment matrix according to the following equations ${ }^{[3]}$.

$$
W_{i}=\frac{\sum_{j=1}^{n} m_{i j}^{\prime}-0.5}{\sum\left[\sum_{j=1}^{n} m_{i j}^{\prime}-0.5\right]}
$$

\section{Analysis and results}

\subsection{Establishment of index and weight of evaluation system}

Questionnaires were sent out to three experts, triangular fuzzy evaluation numbers were sorted out, triangular fuzzy judgment matrix was obtained on average, accurate judgment matrix was obtained according to equation (2), consistency test was carried out, normalized weight vector was calculated, and evaluation system was constructed.

The index aggregate of the evaluation system is as follows: the first level index $\mathrm{A}=\{$ the comprehensiveness of basic data; the authenticity and reliability of traffic volume forecast and analysis data; the design of traffic organization; the function of report; the completeness of report compiling $\}$. The second level index $\mathrm{A}_{1}=$ \{analysis of the current traffic situation around the project; analysis of the planning around the project; situation of important traffic nodes and facilities that have an impact on the project; introduction of construction project\}; $\mathrm{A}_{2}=$ \{investigation, analysis and evaluation of similar projects; prediction of basic data and methods; traffic mode division and assignment; evaluation of external intersection services level $; \quad \mathrm{A}_{3}=$ \{external traffic organization and access type management of the project; location, quantity and width of motor vehicle entrance and exit; garage size, number of entrance and exit, geometric design and management mode; traffic organization and geometric design of internal motor vehicle, non-vehicle and people; external traffic improvement measures and suggestions $\} ; \mathrm{A}_{4}=\{$ the impact of perfecting and improving architectural design;accuracy, rationality and integrity of problems and suggestions $\} ; \mathrm{A}_{5}=$ \{completeness of text compiling ; completeness of drawing .

According to the questionnaire, taking the first level index as an example, the construction process of the whole evaluation system is elaborated, and the triangular fuzzy judgment matrix is sorted out as follows:

$$
\bar{m}^{\prime}=\left[\begin{array}{ccccc}
(0.5,0.5,0.5) & (0.6,0.65,0.72) & (0.37,0.42,0.47) & (0.46,0.52,0.6) & (0.61,0.66,0.73) \\
(0.28,0.35,0.4) & (0.5,0.5,0.5) & (0.37,0.42,0.5) & (0.47,0.52,0.58) & (0.45,0.5,0.58) \\
(0.53,0.58,0.63) & (0.5,0.58,0.63) & (0.5,0.5,0.5) & (0.57,0.61,0.65) & (0.51,0.57,0.62) \\
(0.4,0.48,0.54) & (0.42,0.48,0.53) & (0.35,0.39,0.43) & (0.5,0.5,0.5) & (0.46,0.53,0.62) \\
(0.27,0.34,0.39) & (0.42,0.5,0.55) & (0.38,0.43,0.49) & (0.38,0.47,0.54) & (0.5,0.5,0.5)
\end{array}\right]
$$

According to equation (2), the exact judgment matrix is obtained as follows:

$$
m^{\prime}=\left[\begin{array}{ccccc}
0.5 & 0.65 & 0.42 & 0.52 & 0.66 \\
0.35 & 0.5 & 0.43 & 0.52 & 0.51 \\
0.58 & 0.57 & 0.5 & 0.61 & 0.57 \\
0.48 & 0.48 & 0.39 & 0.5 & 0.53 \\
0.34 & 0.49 & 0.43 & 0.47 & 0.5
\end{array}\right]
$$

Consistency test was carried out, and $\mathrm{P}=0.072<0.2$ was calculated according to equation (3), which satisfied the consistency and the judgment matrix did not need to be adjusted.

According to equation (4), the normalized weight vector is calculated as follows:

$$
W=\{0.23,0.18,0.23,0.19,0.17\} \text {. }
$$

\subsection{Establishment of Evaluation Model}

According to the above indexes and weight distribution, the weight vectors of second level indexes are calculated in the same way, and the corresponding weight vectors of the evaluation system is determined as follows:

$$
\begin{aligned}
& W=\{0.23,0.18,0.23,0.19,0.17\}, \\
& W_{1}=\{0.25,0.24,0.28,0.23\}, \\
& W_{2}=\{0.32,0.22,0.23,0.24\}, \\
& W_{3}=\{0.22,0.21,0.19,0.19,0.19\}, \\
& W_{4}=\{0.57,0.43\}, W_{5}=\{0.53,0.47\}
\end{aligned}
$$

\subsection{Application Method of Evaluation System}

When applying the evaluation system to evaluate a traffic impact evaluation report, it is necessary to classify the evaluation firstly. Generally, the evaluation is divided into four categories: $\mathrm{V}=$ \{excellent, good, qualified, unqualified $\}$. Through consultation from expert, 100-90 is classified as excellent, 89-75 is classified as good, 7460 is classified as qualified, 59-0 is classified as unqualified. The rating vector $\mathrm{H}=\{1,0.8,0.5,0.3\}$ is obtained by quantifying the rating of the evaluation with a 1-point system ${ }^{[9]}$.

Establish a group of assessors for evaluation: each member evaluates report according to each second level index, and finally counts the people number of each second level index with each rating grade. The 
evaluation results are transformed into a fuzzy evaluation matrix: taking the number of people in each grade as a molecule and the total number of assessors as denominator, the dimensionless number less than 1 is obtained to form the evaluation matrix. For example, a certain index has 2 excellent grades, 2 good grades, 1 qualified, and the rest is 0 . That is to say, the evaluation vector of the improved index is $\{0.4,0.4,0.2,0\}$.

By multiplying the established fuzzy evaluation matrix with the corresponding weight vector of the evaluation system, the first-level evaluation vector B can be obtained. According to the principle of maximum membership degree ${ }^{[10]}: \max \left(b_{j}\right)=b_{k}$, the evaluation grade will be evaluated as $b_{k}$. Further calculation of the comprehensive evaluation score: According to the selected evaluation vector, $\mathrm{H}=\{1,0.8,0.5,0.3\}$, After weighted average of the comprehensive evaluation result $\mathrm{B}$, the total score $\mathrm{M}$ can be obtained, and the evaluation object can be ranked by the score.

$$
\mathrm{M}=\mathrm{B} \cdot \mathrm{H}^{\mathrm{T}}=\left(b_{1}, \cdots, b_{n}\right)\left[\begin{array}{l}
h_{1} \\
\cdots \\
h_{n}
\end{array}\right]
$$

6. Xia C.H., Liu J.X. (2010). Selection of Evaluation Period for Traffic Impact Assessment of Construction Projects. Science \& Technology Information, (18):226-227.

7. Cheng C., Liao J.X., Zhu X.M. (2008). CRBT QoS evaluation based on triangular fuzzy number analytic hierarchy process. Journal of Chongqing University of Posts and Telecommunications(Natural Science Edition),20(1):94-99.

8. Xiao Y., Li H. (2003). Improvement on Judgement Matrix Based on Triangle Fuzzy Number. Fuzzy Systems and Mathematics, 17(2):59-64.

9. Rao X., Huang H.L. (2003). Studies and Application on Evaluation Index System for the Stow Net Operation Performance via the Vague APH Method. Journal of Agricultural Science and Technology, 18( 5) : 207-214.

10. Zhou Z. Y. (2013) Construction of dynamic evaluation index system for scientific and technological achievement. Technol. Innov. Manag., 34(5): $430-433$.

\section{Discussion}

The accuracy of the evaluation system based on triangular fuzzy number method depends on the number, points of view and professional condition of experts. In this study, considering the consultation questionnaire data, experts give more neutral opinions, especially reflected in the weight of several second level indexes. In the future, experts will be divided into management, traffic experts and so on. They will consult and answer questions from different perspectives in order to reflect the focus of traffic impact evaluation reports, and the evaluation system will be more accurate.

\section{References}

1. Zheng L. Y, Jin L.Y., Zheng S. (2013) Transportation impact analysis research review and prospect. Planners, 29(07):5-9.

2. Hou C.M., Jin L.Y. (2018). Main problems and reflections on current traffic impact evaluation. Low Carbon World,(07):292-293.

3. Yue L.H., Zhang G.X., Jiang Y. (2019). Research on construction safety management based on triangle fuzzy number analytic hierarchy process. Value Engineering,38(24):75-78.

4. Huo Y.M., Chen J., Li X.H. (2012). Post-evaluation Model of Traffic Impact of Urban Construction Projects. Journal of Transportation Engineering, 12(01):79-86.

5. Qian J., Yuan C.L., Yang L.L. (2012). Comparison of Methods for Determining Traffic Impact Range. Transportation Technology and Economy, 14(01):4548. 